\title{
Terminal Design of Nontouch Queryable Photoelectric Pulse Oximetry
}

\author{
Lan $\mathrm{Ju}^{1}$, a , Qingbin Meng, \\ ${ }^{1}$ College of Electronic Information and Optical Engineering, Nankai \\ University, Tianjin, China \\ ${ }^{2}$ Nankai University Binhai College, No.634, Xuefu road, Dagang, Tianjin, \\ China \\ ankjulan@126.com, ${ }^{b}$ bhmengqb@nankai.edu.cn
}

\begin{abstract}
.
Aimed at monitoring dynamic human pulse and blood oxygen saturation for physical fitness, health care and other fields, a nontouch queryable photoelectric pulse oximetry terminal was designed. The terminal uses a transmission type oxygen probe to collect information of pulse and blood oxygen saturation, uses a low power consumption, low cost STC12C5A16AD microcontroller for analysis and processing of these achieved information, and uses a nRF2401 wireless communication chip for construction of nontouch queryable communication mechanism. The users in the gym or nursing home can query up to 255 terminals and get the real-time monitoring information. Through the experiments, this terminal can effectively provide pulse and blood oximetry saturation monitoring data, can meet the daily physical fitness, health care and other non medical application requirements
\end{abstract}

Keywords: oxygen saturation;photoelectric pulse oximetry;wireless communication

\section{Introduction}

Pulse and blood oxygen saturation is an important physiological index, can effectively and directly reflect the physiological state of human respiratory and circulatory system ${ }^{[1][2]}$. At present, finger type non-invasive photoelectric pulse oximeter monitor has been widely used in the surgery , anesthesia, intensive care and other medical fields ${ }^{[3][4]}$. 
In recent years, with the development of economy and society, the needs and requirements of physical fitness and health care are increasing continuously. For fitness and health care applications, pulse and blood oxygen saturation monitoring terminal should have the advantages of portability, while also supporting the fitness coach, nursing staff can nontouch real-time query multiple monitoring terminals.

A photoelectric pulse oximetry monitoring terminal is designed in this paper.This terminal has the advantages of portability. Query center can carry out nontouch query with up to 255 monitoring terminals. This designed monitoring terminal can be widely applied to the gym, nursing home, health care centers etc..

\section{Design of Monitoring System}

Structure of monitoring system. The monitoring system consists of two kinds of devices, which are query center and monitoring terminal. To the gym as an example, the fitness coach uses the query center, for getting pulse and blood oxygen saturation monitoring data of his students. Monitoring terminal is placed on the student body, collecting the real-time information of pulse and blood oxygen saturation. The query center and the monitoring terminal transmit data to each other through the wireless communication channel. Fitness coaches give query instructions to a monitoring terminal through the query center, and collect the real-time data from this corresponding monitoring terminal. Coaches can also view all the history record stored in the query center.

Monitoring terminal. The main task of monitoring terminal is collecting pulse and blood oxygen saturation information and making data proceeding. When the query center issued query instruction, the monitoring terminal uploads these processed data to the query center. Therefore, the monitoring terminal mainly consists of five functional modules. The five function modules are photoelectric sensing module, analog signal processing module, core control module, wireless 
communication module and power supply module. Structure of monitoring terminal is shown in Fig. 1.

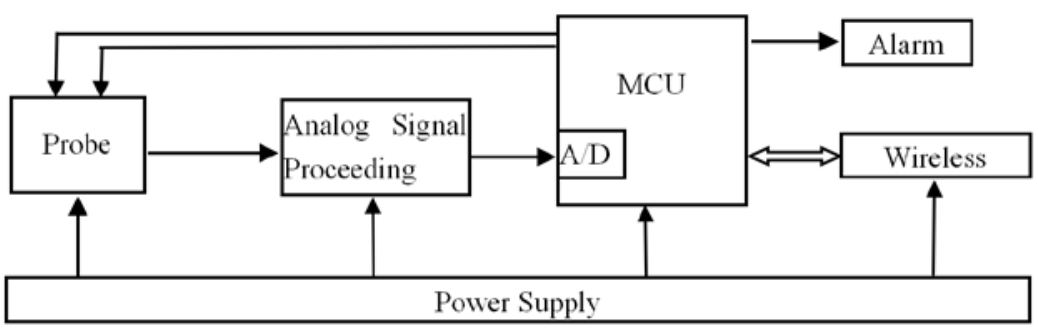

Fig. 1 Structure of monitoring terminal

Principle of blood oxygen saturation detection. Blood oxygen saturation $\left(\mathrm{SpO}_{2}\right)$

is determined by the content of oxygenation hemoglobin $\left(\mathrm{HbO}_{2}\right)$ and deoxygenated hemoglobin $(\mathrm{Hb})$ in arterial bloodstream. Photoelectric transmittance oximetry is a noninvasive detection method ${ }^{[5]}$. According to the phenomenon that the absorption coefficients of $\mathrm{HbO}_{2}$ and $\mathrm{Hb}$ to red light and infrared light are different, this method uses the Lambert-Beer law to calculate $\mathrm{SpO}_{2}$. The general used formula is:

$$
\mathrm{SpO}_{2}=\frac{C_{\mathrm{HbO}_{2}}}{C_{\mathrm{HbO}_{2}}+C_{\mathrm{Hb}}}
$$

In formula (1), $C_{\mathrm{HbO}_{2}}$ is the concentration of $\mathrm{HbO}_{2}$ in arterial blood, $C_{\mathrm{Hb}}$ is the concentration of $\mathrm{Hb}$ in arterial blood.

When a monochromatic light with wavelength $\lambda$ and light intensity $\mathrm{I}_{0}$, vertical irradiated to the finger, in the conduction path, the monochromatic light will be absorbed both by the pulsatile arterial blood and other non pulsating tissue (such as skin, muscle, soft tissue, bone, venous blood etc.) , light intensity of the transmitted light is made up of two part, DC component and AC component. The DC component indicates the light absorption of all the non fluctuating tissue, while the AC component indicates the light absorption of the $\mathrm{HbO}_{2}$ and $\mathrm{Hb}$ in arterial blood. 
With the arterial pulse, light conducting path length will change. Assuming the conduction path length changes $\Delta L$, the transmitted light intensity corresponded changes $\Delta I$, According to the Lambert-Beer law, the transmitted light intensity can be expressed as:

$$
I+\Delta I=I_{0} e^{-\varepsilon_{0} C_{0} L} e^{-\varepsilon_{H b O_{2}} C_{H b O_{2}}(L+\Delta L)} e^{-\varepsilon_{H b} C_{H b}(L+\Delta L)}
$$

$\varepsilon_{0}$ is the total absorption coefficient of all the non fluctuating tissue, $C_{0}$ is the concentration of light absorbing materials, $L$ is she light conducting path length; ${ }_{\mathrm{HbO}_{2}}$ and $\varepsilon_{\mathrm{Hb}}$ are respectively absorption coefficients of $\mathrm{HbO}_{2}$ and $\mathrm{Hb}$ in arterial blood. Because $\Delta I / I \ll 1$, So:

$$
\ln \left(\frac{I+\Delta I}{I}\right)=-\left(\varepsilon_{\mathrm{HbO}_{2}} C_{H b O_{2}}+\varepsilon_{H b} C_{H b}\right) \Delta L \approx \frac{\Delta I}{I}
$$

In practice, red light with wavelength $660 \mathrm{~nm}$ and infrared light with wavelength $940 \mathrm{~nm}$ are often used as the incident light source. When two monochromatic lights with these two kinds of specific wavelengths vertical irradiated to the finger, according to formula (3), we can obtain:

$$
\frac{\Delta I_{660} I_{940}}{\Delta I_{940} I_{660}}=\frac{\varepsilon_{\mathrm{HbO}_{2}}^{660} C_{\mathrm{HbO}_{2}}+\varepsilon_{\mathrm{Hb}}^{660} C_{\mathrm{Hb}}}{\varepsilon_{\mathrm{HbO}_{2}}^{940} C_{\mathrm{HbO}_{2}}+\varepsilon_{\mathrm{Hb}}^{940} C_{\mathrm{Hb}}}
$$

$\frac{\Delta I_{660} I_{940}}{\Delta I_{940} I_{660}}$

$\Delta I_{940} I_{660}$ is called the characteristic value, denoted by R. Feed R into formula (4) , $C_{\mathrm{HbO}_{2}}$ and $C_{\mathrm{Hb}}$ can be obtained; then the results are applied to formula (1), we get the formula for the calculation of blood oxygen saturation: 


$$
S p O_{2}=\frac{\varepsilon_{\mathrm{Hb}}^{660}-\varepsilon_{\mathrm{Hb}}^{940} R}{\varepsilon_{\mathrm{Hb}}^{660}-\varepsilon_{\mathrm{HbO}_{2}}^{660}+\left(\varepsilon_{\mathrm{Hb}}^{940}-\varepsilon_{\mathrm{HbO}_{2}}^{940}\right) R}
$$

$\varepsilon_{\mathrm{Hb}}^{660}, \varepsilon_{\mathrm{HbO}_{2}}^{660}, \varepsilon_{\mathrm{Hb}}^{940}, \varepsilon_{\mathrm{HbO} \mathrm{O}_{2}}^{940}$ are constants, can be obtained by the spectral analysis method. Then there exists a corresponding relation between $\mathrm{SpO}_{2}$ and $\mathrm{R}$. Through the photoelectric sensor technique to obtain $I_{660}, \Delta I_{660}, I_{940}, \Delta I_{940}$, calculate $\mathrm{R}$, and then calculate $\mathrm{SpO}_{2}$.

\section{Design and implementation of monitoring terminal}

Photoelectric probe. Monitoring terminal uses a 7 pins adult finger type photoelectric probe. The probe contains a bidirectional driving dual wavelength light emitting diode, can produce a red light with wavelength $660 \mathrm{~nm}$ and an infrared light with wavelength $940 \mathrm{~nm}$. There is a photodiode in the probe to collect transmitted light, and transform the light into current signal. Structure of the photoelectric probe is shown in Fig. 2.

Monitoring terminal adopts $\mathrm{H}$ Bridge driving circuit to drive the probe bidirectional driving dual wavelength light emitting diode. The microcontroller provides driving control signals to the $\mathrm{H}$ bridge circuit, makes the light emitting diode alternately producing $660 \mathrm{~nm}$ red light and $940 \mathrm{~nm}$ infrared light. The probe photodiode operates in time-division multiplexing mode, for realization of the two optical signal acquisition.

Analog signal processing. The current signal produced by the photoelectric diode in photoelectric oxygen probe is very small. This analog signal generated by the probe must get appropriate treatment before the useful signal was extracted from noise and interference. Analog signal processing includes $\mathrm{I} / \mathrm{V}$ convert, amplifier and filter. The signal processing flow is shown in Fig. 3. 


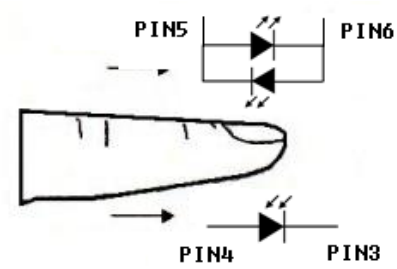

Fig. 2 structure of probe

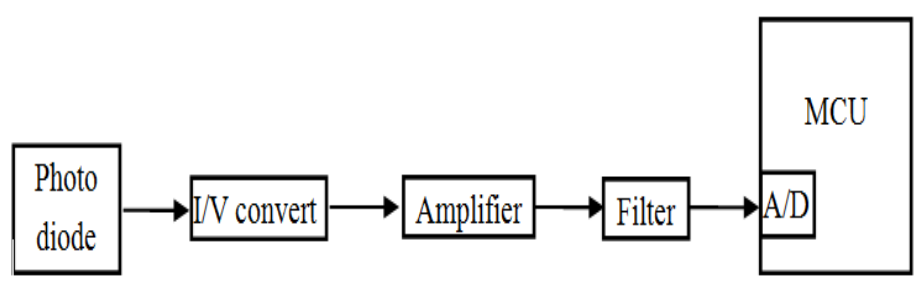

Fig. 3 structure of analog signal processing

Cause the current signal generated by the photodiode is very small, the I/V convert circuit requires high input impedance, low noise, low offset current. Monitoring terminal adopts AD795 operational amplifier to realize the current to voltage conversion. Amplifier and filter circuit adopts LM324 operational amplifier, to realize 200 times voltage amplifier and low pass filter.

Main control module. Use the low power, low cost STC12C5A16AD microcontroller as the main control chip. The MCU has on-chip 10 bit A/D conversion circuits, so, the proceeded analog signal inputs the microcontroller directly. MCU program provides driving control signals to the $\mathrm{H}$ bridge circuit, to control the light emitting diode alternately producing $660 \mathrm{~nm}$ red light and 940 $\mathrm{nm}$ infrared light. And control the A/D conversion circuit to convert the useful signal during the light emitting diode producing a certain wavelength light.

The main control program of monitoring terminal mainly complete the tasks of the red light producing, red light corresponded sensing information collection, infrared emission, infrared light corresponded sensing information collection, receiving a query command from the query center and giving response, monitoring abnormal pulse and blood oxygen saturation information and send out sound or light alarm. 
Wireless communication. Wireless data communication module of monitoring terminal adopts nRF2401 wireless transceiver chip. Communication occupies on 2.4GHz ISM frequency band.

\section{The results of Experiment}

Monitoring terminals collect human pulse and blood oxygen saturation information. Query center gets the pulse and blood oxygen data through wireless communication. Analysis the measurement results of the monitoring terminal and the measurement results of a certain type of medical pulse oximetry, in order to figure out whether the designed terminal has good accuracy and stability. Experiments were carried out with 10 adult men and 10 adult women, the measurement results are shown in Fig. 4 and Fig. 5.

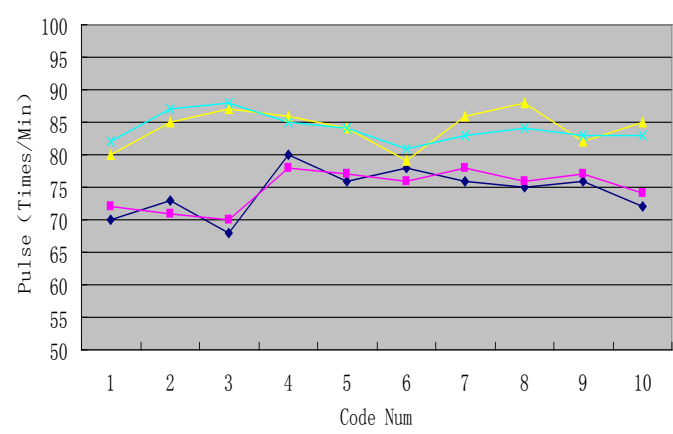

$\multimap$ Terminal (M) $₫-$ Medical Equ(M) $\_$Terminal $(\mathrm{F})-$-Medical Equ(F)

Fig. 4 pulse curves

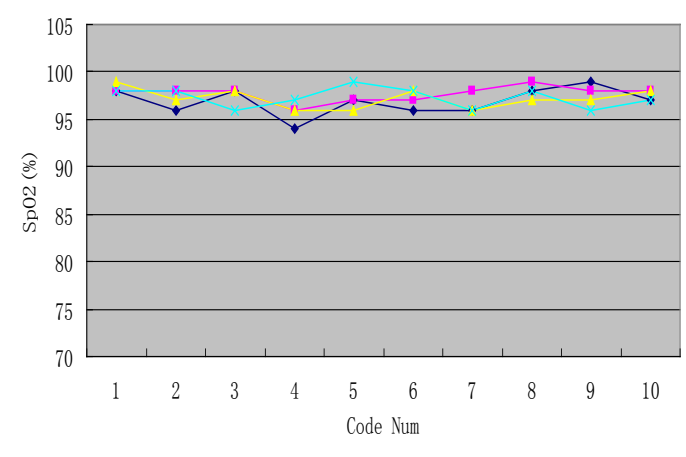

- Terminal (M) - Medical Equ(M) + Terminal (F) $-x$ Medical Equ(F)

Fig. 5 oxygen saturation curves 
According to the results, the designed monitoring terminal, compared with a certain type of medical equipment, can provide basically credible pulse and blood oxygen saturation data.

\section{Conclusion}

A nontouch queryable photoelectric pulse oximetry terminal was designed in this paper. The terminal uses a transmission type oxygen probe to collect information of pulse and blood oxygen saturation, uses a low power consumption, low cost STC12C5A16AD microcontroller for analysis and processing of these achieved information, and uses a nRF2401 wireless communication chip for data transmission between the query center and the terminals. The users in the gym or nursing home can query up to 255 terminals and get the real-time monitoring information. Through the experiments, this terminal can effectively provide pulse and blood oximetry saturation monitoring data, can meet the daily physical fitness, health care and other non medical application requirements.This nontouch queryable photoelectric pulse oximetry monitoring terminal has good application prospect.

\section{References}

[1] Liu Guangwei, Mao Luhong, Xie Sheng, Meng Qingbin, Zhang Qingrong: Journal of Optoelectronics • Laser,Vol.25,No.3, 2014, p.620-624.

[2] YIN Guang-zhi, XING Da, YANG Si-hua: Journal of Applied Physics, 2009, 106(1), p.1186-1193.

[3] Castillejo P, Martinez J F, Rodriguez-Molina J: Wireless Communications, IEEE, 2013,20(4): p.38-49.

[4] Tavakoli M, Turicchia L, Sarpeshkar R: IEEE transaction on biomedical circuits and systems, 2010, 4(1): p.27-38.

[5] Hu Jing, Wang Cheng, Li Zhangjun: Journal of Optoelectronics • Laser,Vol.23,No.8,Mar.2012, p.1631-1624. 